Reprod. Nutr. Dévelop., 1986, 26 (1 B), 369-370.

\title{
Utilisation des substrats glucoformateurs par les hépatocytes isolés de mouton
}

\author{
C. DEMIGNÉ, P. FAFOURNOUX, C. RÉMÉSY, C. YACOUB
}

I.N.R.A., Theix 63122 Ceyrat, France.

Summary. Isolated hepatocytes were prepared from sheep starved for $24 \mathrm{~h}$. At substrate concentrations between 0.5 and $2.0 \mathrm{mM}$, sheep hepatocytes utilized more propionate than butyrate. Other potential glucogenic substrates (lactate, alanine, glutamine) were poorly utilized in basal conditions.

Chez les espèces de laboratoire, des progrès considérables dans la compréhension du métabolisme hépatique ont été obtenus grâce à l'utilisation d'hépatocytes isolés. Des adaptations de la technique de perfusion avec de la collagénase ont permis d'isoler également des hépatocytes de ruminants (Pogson et al., 1984). Nous avons donc mis en œuvre cette méthode pour étudier l'utilisation des principaux substrats de la néoglucogenèse et de l'uréogenèse.

Matériel et Méthode. Des moutons d'environ $40 \mathrm{~kg}$ à jeun $24 \mathrm{~h}$, de race Limousine, sont sacrifiés à l'abattoir sans anesthésie. Après ablation rapide du foie (1 à $2 \mathrm{~min}$ ), le lobe caudé est sectionné et perfusé. II est ensuite transporté sous perfusion dans une enceinte thermostatée à $38^{\circ} \mathrm{C}$. Le débit de la perfusion par du tampon Krebs bicarbonate $\left(\mathrm{pH} \mathrm{7,4)}\right.$ à $38^{\circ} \mathrm{C}$, est de 4 à $5 \mathrm{ml} / \mathrm{min} / \mathrm{g}$ de foie. La perfusion comporte 3 phases successives : 1) rupture des desmosomes à la suite de chélation du $\mathrm{Ca}^{++}$par de I'EGTA; 2) rinçage avec du tampon Krebs ; 3) action de la collagénase $(0,1 \%)$ en présence de $\mathrm{Ca}^{+}+2 \mathrm{mM}$; dans cette dernière étape, le perfusat est recyclé et soumis à un gazage au carbogène. Le tissu est dilacéré au bout de $30 \mathrm{~min}$. Les hépatocytes récoltés sont rincés et purifiés par 3 centrifugations lentes. Leur viabilité estimée par la biréfringence au microscope à contraste de phase est supérieure à $90 \%$. En adaptant le temps de perfusion de la collagénase (25-35 $\mathrm{min})$, la préparation des hépatocytes est réussie 9 fois sur 10 environ.

La disparition des substrats a été appréciée en présence de $30 \mathrm{mg}$ de cellules (poids frais) $/ \mathrm{ml}$ incubées sous atmosphère de carbogène. Les AGV ont été dosés par chromatographie en phase gazeuse et les autres substrats par des méthodes enzymatiques standard.

Résultats et discussion. Utilisation des substrats (tabl. 1). - Elle est appréciée par leur disparition du milieu après 20 et 40 min d'incubation. L'acétate est très faiblement utilisée même pour des concentrations élevées ; par contre le propionate et le butyrate disparaissent rapidement. L'utilisation du propionate est plus de 2 fois supérieure à celle du butyrate. La très faible utilisation de l'acétate provient sans doute de la faible activité de l'acétyl CoA synthétase dans le foie des ruminants (Ricks et Cook, 1981). A la différence du propionate, le lactate $(2 \mathrm{mM})$ est très faiblement utilisé dans les préparations d'hépatocytes isolés $\left(0,10 \mu\right.$ moles $\left.\mathrm{mn}^{-1} \mathrm{~g}^{-1}\right)$. Les vitesses de disparition de l'alanine et de la glutamine sont faibles mais mesurables (respectivement de 0,20 et $0,32 \mu$ moles $\mathrm{mn}^{-1} \mathrm{~g}^{-1}$ ). 
L'ammoniac $(5 \mathrm{mM})$, en présence de $0,25 \mathrm{mM}$ d'ornithine, est bien utilisé $\left(0,69 \mu\right.$ moles $\left./ \mathrm{mn}^{-1} \mathrm{~g}^{-1}\right)$ et la présence de propionate ou de lactate $(2 \mathrm{mM})$ accroît son utilisation (respectivement 1,19 et $1,04 \mu$ moles $/ \mathrm{mn}^{-1} \mathrm{~g}^{-1}$ ).

TABL. 1. - Comparaison de l'utilisation des acides gras volatils par des hépatocytes isolés de mouton.

\begin{tabular}{lccc} 
& $0,5 \mathrm{mM}$ & $1,0 \mathrm{mM}$ & $2,0 \mathrm{mM}$ \\
\hline Acétate & $0,03 \pm 0,01$ & $0,08 \pm 0,02$ & $0,17 \pm 0,02$ \\
Propionate & $0,50 \pm 0,03$ & $0,95 \pm 0,06$ & $1,60 \pm 0,10$ \\
Butyrate & $0,20 \pm 0,02$ & $0,38 \pm 0,02$ & $0,70 \pm 0,04$ \\
\hline
\end{tabular}

Les résultats (vitesse de disparition des substrats du milieu d'incubation) sont exprimés en $\mu$ moles $/ \mathrm{mn} / \mathrm{g}$ d'hépatocytes et représentent les moyennes \pm SEM pour 4 expériences.

Etude de la g/ucogénicité des divers substrats (tabl. 2). - Les hépatocytes de ruminant présentent une très faible utilisation du lactate pour la synthèse de glucose. Le propionate constitue de loin le substrat glucoformateur le plus efficace. L'alanine et la glutamine sont faiblement glucogéniques par rapport au propionate. La différence entre propionate et lactate provient soit d'une trop faible transformation du lactate en pyruvate, soit d'une conversion très lente du pyruvate en oxaloacétate. Le propionate est par contre très rapidement activé (Ricks et Cook, 1981) et sa conversion en glucose est indépendante de l'activité de la pyruvate carboxylase. L'uréogenèse à partir de l'ammoniac $(5 \mathrm{mM})$ est stimulée par le propionate et le lactate $(2 \mathrm{mM}): 0,45$ et $0,55 \mu$ moles $/ \mathrm{mn}^{-1} \mathrm{~g}^{-1}$ respectivement, contre 0,35 chez le témoin. Il pourrait y avoir, chez le ruminant comme chez le monogastrique, un certain couplage entre la néoglucogenèse à partir du lactate et l'uréogenèse à partir de $\mathrm{NH}_{3}$.

TABL. 2. - Effets des différents substrats g/ucoformateurs sur la production de g/ucose ( $\mu$ moles $/ \mathrm{mn} / \mathrm{g}$ poids frais \pm SEM, pour 4 lots d'hépatocytes).

\begin{tabular}{lcc} 
Substrats & $\begin{array}{c}\text { Production } \\
\text { totale }\end{array}$ & $\begin{array}{c}\text { Production nette due } \\
\text { aux substrats étudiés }\end{array}$ \\
\hline Aucun & $0,18 \pm 0,02$ & - \\
Propionate $1 \mathrm{mM}$ & $0,48 \pm 0,03$ & 0,30 \\
Propionate $2 \mathrm{mM}$ & $0,72 \pm 0,04$ & 0,54 \\
L-lactate 2 $\mathrm{mM}$ & $0,26 \pm 0,02$ & 0,08 \\
L-alanine 2 $\mathrm{mM}$ & $0,34 \pm 0,02$ & 0,16 \\
L-glutamine $1 \mathrm{mM}$ & $0,35 \pm 0,03$ & 0,17 \\
\hline
\end{tabular}

En conclusion, le cycle de Cori semble moins important chez les ruminants que chez les monogastriques bien que l'utilisation du lactate soit sans doute plus élevée in vivo qu'in vitro. Par rapport aux hépatocytes isolés de rat qui utilisent préférentiellement le butyrate au propionate, ceux de mouton utilisent mieux le propionate que le butyrate. La technique des hépatocytes isolés devrait permettre une meilleure compréhension des relations entre l'uréogenèse et la néoglucogenèse; par ailleurs l'emploi d'hépatocytes est pratiquement indispensable pour aborder les problèmes de transport membranaire.

Pogson C. I., Carpenter W. R., Cook J. S., Fisher M. J., Lomax M. A., Salter M., Stanley J. C., 1984. A critical approach to the use of isolated liver cells for the study of metabolic events. Proc. Nutr. Soc., 43, 119-132.

Ricks C. A., Cook R. M., 1981. Regulation of volatile fatty acid uptake by mitochondrial acyl CoA synthetase of bovine liver. J. Dairy Sci., 64, 2324-2335. 